\title{
Decreased sleep quality and increased sleep related movements in patients with Tourette's syndrome
} S Cohrs, T Rasch, S Altmeyer, J Kinkelbur, T Kostanecka, A Rothenberger, E Rüther,
G Hajak

\begin{abstract}
Objective-Sleep quality and movement patterns across sleep stages in patients with Tourette's syndrome were examined to determine the influence of syndrome severity on sleep quality and the differential effect of sleep stages on tic and non-tic movements.
\end{abstract}

Methods-Twenty five patients with Tourette's syndrome (mean age 29 (SD 7) years) and 11 control subjects (29 (5) years) were studied by polysomnography and simultaneous split screen video monitoring to record standard sleep variables as well as to evaluate movements to differentiate between tics and regular movements. Severity of Tourette's syndrome during the day was assessed with the Tourette's syndrome severity scale.

Results-Sleep was significantly more disturbed in patients with Tourette's syndrome than in controls, with decreased sleep efficiency and slow wave sleep percentage, increased sleep latency, percentage of stage $I$, percentage of awakeness, number of awakenings, and sleep stage changes and more overall movements during sleep. Severity of Tourette's syndrome during the day correlated significantly and positive with number of awakenings and sleep stage changes and negatively with sleep efficiency. In addition to an increased number of regular movements patients had tics in all sleep stages. Tic frequency as well as frequency of regular movements was significantly higher in REM than in non-REM sleep which was also the case for regular movements of the controls. No disturbance of either REM sleep percentage or REM latency was found.

Conclusion-Despite normal total sleep time and unaltered REM sleep variables patients with Tourette's syndrome have markedly disturbed sleep. Severity of the syndrome during the day is an important predictor of sleep alteration in patients. The increased rate of tics during REM sleep parallels the overall increased movement activity of patients during REM as well as non-REM sleep. The increased motor activity may be attributable to a state of hyperarousal rather than a disturbed cholinergic system.

(F Neurol Neurosurg Psychiatry 2001;70:192-197)

Keywords: Gilles de la Tourette's syndrome; polysomnography; sleep; movement analysis
Gilles de la Tourette's syndrome is a neuropsychiatric disorder that is characterised by multiple motor and vocal tics. It usually starts in childhood and persists in most patients through adulthood with a waxing and waning symptomatology of phenomenologically changing tics of varying severity. Besides other associated symptoms such as coprolalia, attentional deficit disorder, obsessive compulsive traits, echolalia, copropraxia, and echopraxia, up to $60 \%$ of the patients with Tourette's syndrome complain about disturbed sleep. ${ }^{1}$ Most often reported are complaints of insomnia such as difficulty falling asleep or early awakening. ${ }^{2}$

Few polysomnographic studies with varying methodological quality have been undertaken to determine the objective sleep quality of patients with Tourette's syndrome. Conflicting results have been reported concerning an unaffected $^{34}$ or decreased ${ }^{5}$ sleep efficiency, unaffected $^{34}$ or prolonged ${ }^{6}$ sleep latency, increased, ${ }^{4}$ decreased ${ }^{36}$ or unaffected ${ }^{5}$ slow wave sleep, and reduced ${ }^{146}$ or unaffected ${ }^{357}$ rapid eye movement (REM) sleep. No data exist about the influence of Tourette's syndrome severity on objective sleep parameters. Furthermore, the origin and pathophysiology of tic related sleep disturbances has not been unequivocally clarified.

Motor phenomena during sleep in patients with Tourette's syndrome have been interpreted in different ways ranging from REM sleep behaviour disorder ${ }^{8}$ to periodic limb movements. ${ }^{5}$ Earlier studies ${ }^{149}$ reported that tics occur during REM as well as non-REM sleep. However, no data exist on the frequency, intensity, and complexity of tics across sleep stages. Data on tic patterns related to sleep stage are of particular interest to better understand the pathophysiology of Tourette's syndrome. Different neurotransmitter systems have been discussed as being involved in pathophysiology of the disease. Besides the dopaminergic, serotonergic, opioid, and noradrenergic system it has been postulated that the cholinergic system is involved in the pathogenesis of tics. ${ }^{10}$

Considering the aminergic-cholinergic interaction model of sleep regulation ${ }^{11}$ a differential effect of sleep stages on tics and regular (nontic) movements would be expected. Taking into account that the various neurotransmitters involved in the regulation of sleep have been hypothesised to play a major part in the pathophysiology of tics in patients with Tourette's syndrome the aim of this study was to (1) objectively identify sleep quality in patients 
Table 1 Patient characteristics

\begin{tabular}{lllll}
\hline No & Sex & Age & TSSS & Medication $/ 24 h$ \\
\hline 1 & Male & 41 & 8.0 & Tiapride $3 \times 200 \mathrm{mg}$, chloral hydrate $1500 \mathrm{mg}$ \\
2 & Male & 16 & 4.0 & None \\
3 & Male & 25 & 7.0 & None \\
4 & Male & 34 & 4.25 & None \\
5 & Female & 28 & 7.0 & None \\
6 & Male & 39 & 7.5 & Pimozide $2 \mathrm{mg}$ every other day (none before night II) \\
7 & Female & 30 & 7.0 & Carbamazepine $300 \mathrm{mg}$, clomipramine $150 \mathrm{mg}$. \\
8 & Male & 23 & 8.0 & None \\
9 & Male & 25 & 3.0 & None \\
10 & Female & 26 & 5.0 & None \\
11 & Female & 38 & 0.5 & None \\
12 & Female & 27 & 6.5 & Pimozide $6 \mathrm{mg}$, tiapride 200 mg \\
13 & Male & 35 & 3.5 & None \\
14 & Male & 23 & 4.5 & Haloperidol $1 \mathrm{mg}$ \\
15 & Female & 28 & 4.0 & Clozapine $75 \mathrm{mg}$ \\
16 & Male & 37 & 6.75 & None \\
17 & Male & 25 & 5.5 & None \\
18 & Male & 23 & 5.5 & Clomipramine $150 \mathrm{mg}$ \\
19 & Male & 30 & 7.0 & Fluvoxamine $400 \mathrm{mg}$ omipramol $50 \mathrm{mg}$ \\
20 & Female & 19 & 6.5 & Pimozide $300 \mathrm{mg}$ \\
21 & Female & 30 & 6.75 & Clomipramine $225 \mathrm{mg}$, bromperidol $3 \mathrm{mg}$, biperiden $4 \mathrm{mg}$ \\
22 & Male & 24 & 7.0 & Self medication with cannabis \\
23 & Male & 24 & 4.5 & None \\
24 & Female & 30 & 6.0 & None \\
25 & Male & 43 & 6.75 & Pimozide $200 \mathrm{mg}$ \\
\end{tabular}

TSSS $=$ Tourette syndrome severity scale. ${ }^{12}$

with Tourette's syndrome and (2) to determine the influence of severity of Tourette's syndrome on standard polysomnographic variables as well as on the frequency and type of movements exhibited in comparison with healthy subjects in REM and and non-REM sleep.

\section{Methods}

PATIENTS AND CONTROLS

Twenty five patients with Tourette's syndrome (mean age 29.04 (SD 6.8) years, range 16-44; 16 male and nine female) and a group of 14 healthy volunteers (28.7 (SD 5) years, range 23-39; eight male and six female) were studied for comparison of sleep variables. Eleven of these 14 healthy subjects (28.9 (SD 5.2) years, range 24-39 years; seven male and four female) were included in the video analysis of movement variables. Patients were recruited from the outpatient clinic and self help groups where two of the authors (TR and SA) presented the possibility to the participants of being examined polysomnographically within a scientific study. Control subjects were recruited by word of mouth, mostly being university associated students, employees, or friends of these who heard of the study and were interested in participating. A detailed clinical history was taken to ensure current health of the control subjects. All patients fulfilled the diagnostic criteria for Tourette's syndrome according to the International Classification of Diseases, 10 th edition (ICD-10) and the Diagnostic and Statistical Manual, 4th edition (DSM IV). Demographic data of the patients, current medication, and severity of the disease according to the Tourette's syndrome severity scale (TSSS) $)^{12}$ are summarised in table 1 . Severity ranged from 0.5 (mild) to 8.0 (severe) with most of the patients being moderately to severely affected.

The study was approved by the local ethics committee and was conducted according to the declaration of Helsinki. Each subject gave written informed consent after a complete description of the study.
POLYSOMNOGRAPHY

All subjects underwent polysomnographic recordings for two consecutive nights according to standard criteria. ${ }^{1314}$ To exclude first night effects only results of the second night were analysed. Polysomnography included EEG (C3/A2), electro-oculogram, submental EMG, ECG, and EMG of the anterior tibial muscles. Additional superficial EMG leads were placed on the muscle groups exhibiting typical tics during wake time (sternocleidomastoid, trapezius, masseter, and brachioradial muscles). Patients and subjects were allowed to go to bed at their usual bedtime and to get up at their convenience. Sleep was recorded at a paper speed of $10 \mathrm{~mm} / \mathrm{s}$ on a 21 channel polysomnograph (Nihon Kohden, Japan) and a computerised video monitoring system. Sleep was scored according to the standard criteria of Rechtschaffen and Kales ${ }^{13}$ in 30 second epochs. Evaluation of EEG arousals followed criteria of the American Sleep Disorders Association. ${ }^{15}$

\section{MOVEMENT ANALYSIS}

In addition to polysomnographic recordings subjects' movements were monitored with an infrared camera. Illumination was provided by two infrared lighting systems, allowing excellent video recordings in a dark room. Sound was recorded from a microphone located 91 $\mathrm{cm}$ ( 3 feet) above the subject's bed. Split screen video monitoring was used to have the visual image as well as polysomnographic data stored on the same tape. With this technique simultaneous analysis of polysomnographic variables, visual analysis of movements, and evaluation of audio signals was possible.

Patients and subjects spent 2 days in the sleep laboratory. Daytime observation gave an opportunity to the scorers (TR and SA) to become familiar with the individual tics of the patients. Tics were classified using a rating system which followed the DSM-IV definition of a tic as a sudden, rapid, recurrent, non-rhythmic stereotyped movement or vocalisation. Inconclusive scoring of a particular movement was followed by evaluation of this movement by the second scorer and final classification was done after consensus conference. Tic intensity was classified into three levels as: mild (1), moderate (2), and severe (3). Key aspects of tic intensity were strength, impulse, and range of the tic movement. Tic complexity was divided into two categories according to Leckman et $a l^{16}$ : Simple tics consist of purposeless abrupt movements such as grimacing, eye blinking, shoulder shrugging etc. Complex tics were slower movements that look as if they had a purpose such as touching, biting, head banging, itching etc.

Tics and regular movements during sleep of patients and control subjects were scored by analysis of the split screen video recordings. Because of the brief and abrupt nature of tics it was possible to identify and count individual tics; whereas it was often impossible to determine the end of a regular movement and the beginning of the next regular movement. For determination of reliability of movement scoring the concordance rate of the two raters 
Table 2 Sleep variables of patients with Tourette's syndrome $(n=25)$ compared with healthy controls $(n=4)$

\begin{tabular}{llll}
\hline & $\begin{array}{l}\text { Tourette patients } \\
(n=25)\end{array}$ & $\begin{array}{l}\text { Controls } \\
(n=14)\end{array}$ & $p$ Value \\
\hline TIB (min) & $519(81)$ & $486(53)$ & $\mathrm{NS}$ \\
SPT (min) & $472(87)$ & $464(56)$ & $\mathrm{NS}$ \\
TST (min) & $428(93)$ & $446(60)$ & $\mathrm{NS}$ \\
SE (TST in \%TIB) & $82(10)$ & $92(6)$ & $<0.001$ \\
Sleep latency (min) & $40(49)$ & $17(9)$ & $<0.05$ \\
REM latency (min) & $97(62)$ & $94(38)$ & $\mathrm{NS}$ \\
Wake\% (SPT) & $9.6(5.6)$ & $3.9(3.7)$ & $<0.001$ \\
St I\% (SPT) & $11.9(4.1)$ & $8.7(2.4)$ & $\mathrm{NS}$ \\
St II\% (SPT) & $47.1(9.1)$ & $49.4(5.8)$ & $<0.05$ \\
SWS\% (SPT) & $10.2(7.0)$ & $14.7(3.9)$ & $\mathrm{NS}$ \\
REM\% (SPT) & $20.8(4.0)$ & $22.8(4.8)$ & $<0.005$ \\
St I\% (TST) & $13.3(5.1)$ & $9.1(2.9)$ & $\mathrm{NS}$. \\
St II\% (TST) & $51.9(9.0)$ & $51.3(5.8)$ & $<0.05$ \\
SWS\% (TST) & $11.2(7.8)$ & $15.2(3.9)$ & $\mathrm{NS}$ \\
REM\% (TST) & $23(4.5)$ & $23.7(4.9)$ & $<0.001$ \\
Awakenings/h SPT & $2.8(1.6)$ & $1.5(0.7)$ & $<0.05$ \\
Sleep stage changes/h SPT & $18.6(5)$ & $15.8(2.4)$ & \\
& & &
\end{tabular}

Values are means (SD), $t$ test (two tailed); TIB=time in bed; SPT=sleep period time; TST=total sleep time; SE=sleep efficiency =TST/TIB; SL=sleep latency; REM-latency=latency to REM sleep from sleep onset; W\%, St I \%, St II\%, St SWS \%, REM \% (SPT)/(TST)=percentage of wake, stage I, stage II, slow wave sleep, REM sleep of SPT or TST respectively; awakenings/h $\mathrm{SPT}=$ number of awakenings/hour SPT; sleep stage changes/h SPT=sleep stage changes/hour SPT.
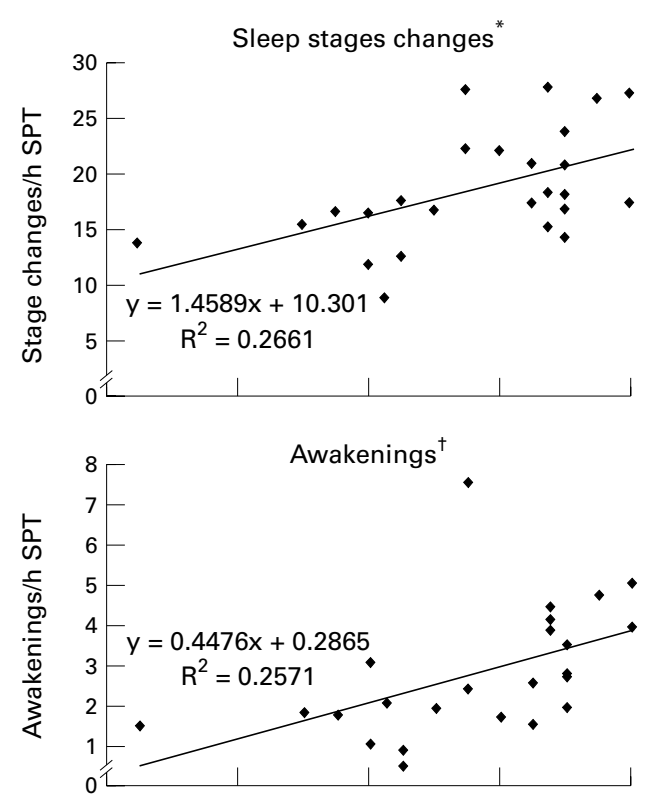

was calculated for identification of tic versus non-tic movement epoch, complexity, and intensity of tic movements. For this purpose a total of 1300 sleep epochs from three different patients were analysed by both raters. The concordance rate for all variables was higher than $85 \%$.

To provide a basis for a comparison of tic and non-tic movements, epochs in which at least one tic or one regular movement occurred were counted and classified as an epoch with a tic, a non-tic movement, or both. A tic and non-tic movement index was calculated for REM and non-REM sleep separately. The index corresponds to the percentage of epochs of a specific sleep stage (REM or non-REM sleep) during which a specific movement (tic or non-tic movement) occured.

STATISTICS

For descriptive statistics of sleep variables, means (SD) were calculated. For comparison of sleep and movement variables between the group of patients and the control group as well as for the comparison between the medicated and unmedicated patients Student's $t$ test (two tailed) was chosen. For further analysis, a Pearson correlation coefficient was calculated to correlate sleep variables and rating scores on severity of Tourette's syndrome. The level of significance for all calculations was set at 5\%.

\section{Results}

SLEEP VARIABLES

Thirteen of the patients with Tourette's syndrome were free of medication for at least 4 weeks. The other 12 patients were taking different medication including neuroleptic and antidepressant drugs (table 1). One patient was included into the group of medicated patients because he medicated himself by frequent application of cannabis.

None of the sleep variables showed a significant difference between medicated and unmedicated patients, which might be due to the fact that treated and untreated patients had tics

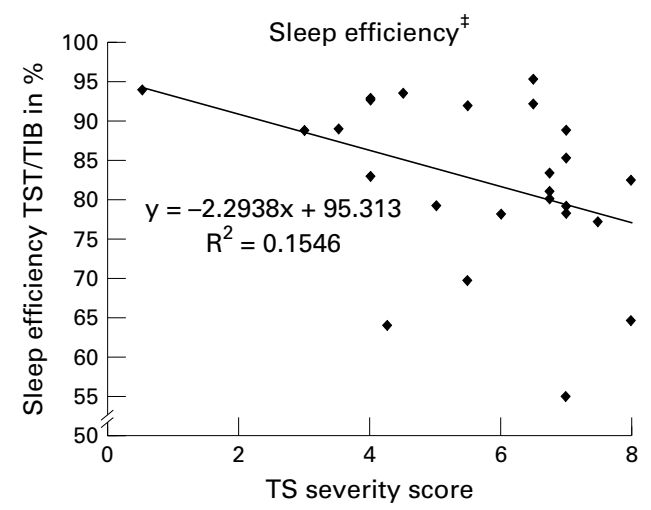

Figure 1 Correlation between Tourette's syndrome severity score and sleep variables $(n=25)$. ${ }^{\star}$ Significant correlation $(r=0.51 ; p<0.01)$; tsignificant correlation $(r=0.52$; $p<0.01)$; $\neq$ significant correlation $(r=-0.39 ; p<0.05)$.

and associated symptomatology to a comparable degree. Therefore, data for the patients were pooled for further analysis. Time in bed, sleep period time (time from first sleep onset to awakening), total sleep time (sleep period time - time awake), percentage sleep stage II of total sleep time, percentage REM sleep of total sleep time (\% REM), and REM latency (time from lights out as well as from sleep onset to first epoch of REM sleep) of the patients with Tourette's syndrome were not significantly different from the control group. However, in comparison with the control subjects (table 2) patients with Tourette's syndrome showed significantly reduced sleep efficiency (total sleep time/time in bed) and percentage of slow wave sleep, significantly prolonged sleep latency, significantly increased percentage of sleep stage I, percentage of time awake, and increased number of awakenings and sleep stage changes/ hour sleep period time.

A significant correlation between Tourette's syndrome severity score and number of awakenings/hour, number of sleep stage changes during sleep period time, and sleep efficiency was found (fig 1). 


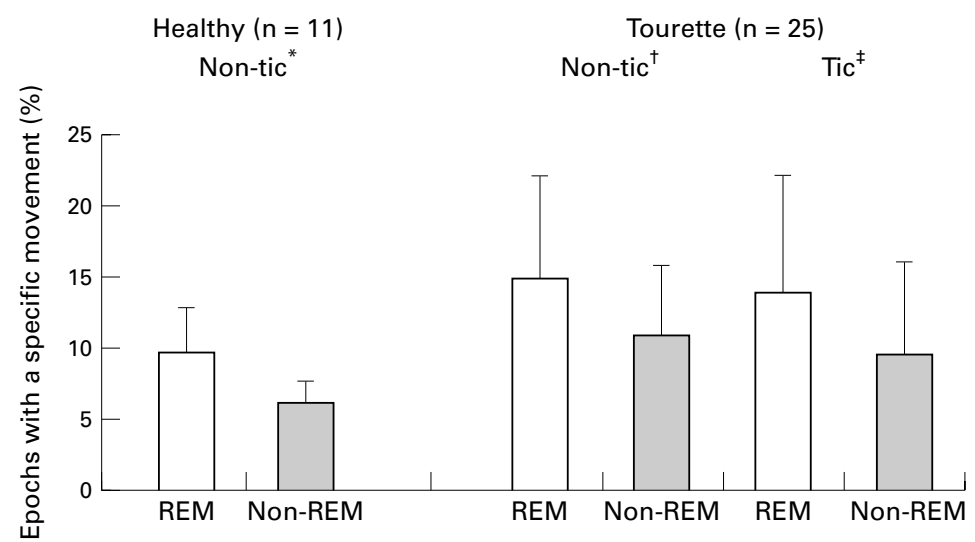

Figure 2 Frequency of tic and non-tic epochs in patients with Tourette's syndrome $(n=25)$ and healthy controls $(n=11)$ in percentage of epochs of REM or non-REM sleep.

*Significant sleep stage effect (10 (3) $v 6$ (2); $p<0.01)$; †sgnificant sleep stage effect (15

(7) $v 11$ (5); $p<0.02)$; ¥significant sleep stage effect (14 (8) $\vee 9(6) ; p<0.02)$.

MOVEMENT ANALYSIS

Patients with Tourette's syndrome versus healthy subjects

Patients with Tourette's syndrome showed a significantly higher movement index for total sleep time than healthy subjects (17 (7) $v 7$ (1); $\mathrm{p}<0.0001)$. This difference was found in non-REM sleep (16 (8) v 6 (2); p<0.0001) as well as in REM sleep (21 (10) v 10 (3); $\mathrm{p}<0.0001)$. Patients not only showed significantly more overall movements including tics during sleep but had a higher non-tic movement index compared with healthy controls (12 (5) $v 7$ (1); $<<0.0001$ ) as well. This was the case for REM sleep $(\mathrm{p}<0.01)$ as well as for non-REM sleep ( $\mathrm{p}<0.001$, fig 2$)$.

Patients with Tourette's syndrome showed a similar number of wake epochs after a tic or a non-tic movement as control subjects. However, epochs with either a tic movement, a nontic movement, or both in patients were on average significantly less often associated with EEG arousals than movement epochs of control subjects (78.0\% (14.1\%) v 94.6\% (8.7\%); $\mathrm{p}<0.001\}$.

\section{Healthy subjects}

Comparing the movement frequency in REM and non-REM sleep healthy subjects had a higher movement index in REM than in non-REM sleep ( $<<0.01$, fig 2 ).

Patients with Tourette's syndrome

On average 184.4 (151.8) tics were found during total sleep time. This corresponded to 0.46 (0.41) tics every minute. Tics were found in all patients in sleep stage I, stage II, and REM sleep and in 20 of the 22 patients reaching slow wave sleep. Similar to the results of healthy subjects, patients with Tourette's syndrome showed a significantly higher movement index during REM than during non-REM sleep $(\mathrm{p}<0.02)$; the same was true for tics: there was a significantly increased tic index during REM compared with non-REM sleep $(p<0.02$, fig 2).

Tics showed a higher intensity in REM than in non-REM sleep (1.37 (0.07) v 1.21 (0.14); $\mathrm{p}<0.002)$ whereas tic complexity was not different between REM and non-REM sleep.
There was no significant differential effect of tics versus non-tic movements in consecutive epochs of wake after a tic or non-tic movement. However, epochs with only tics showed significantly less often EEG arousals than epochs with non-tic movements (55.4\% (17.1\%) $v$ $73.8 \%(18.3 \%) ; \mathrm{p}<0.001)$. If both tics and non-tic movements occurred in one epoch there were significantly more often EEG arousals in comparison with either only tics or only non-tic movements (88.0\% (19.8\%) v $55.4 \% \quad(17.1 \%), \mathrm{p}<0.01 ; 73.8 \% \quad(18.3 \%)$; $\mathrm{p}<0.01$ respectively\} .

\section{Discussion}

SLEEP VARIABLES

Patients with Tourette's syndrome showed a markedly altered sleep quality with difficulties initiating and maintaining sleep. They had significantly longer sleep latency, reduced sleep efficiency, more percentage stage I, higher frequency of sleep stage changes as well as awakenings along with a reduced slow wave sleep percentage. The comparison with data from other studies is only partially possible because most of the authors have not provided essential information about basic polygraphic variables including total sleep time. ${ }^{41719}$ The studies providing this polygraphic information examined only very small groups of patients ${ }^{53}$ (two to seven) and lacked details about the time of determining the end of the polygraphic registration. This might be of relevance because of the circadian regulation of REM sleep propensity with more REM sleep in the morning hours. ${ }^{18}$ Patients and subjects in our laboratory were allowed to sleep at the time of their convenience including spontaneous awakening in the morning. Thereby the patients with Tourette's syndrome might have been able to compensate for their lost sleep during the night, which would explain the unaltered total sleep time in comparison with the healthy controls in our study. The unchanged REM percentage together with the unaltered REM latency support the hypothesis of a stable and unaffected cholinergic system in Tourette's syndrome.

The two aminergic transmitters serotonin and noradrenaline (norepinephrine) have been found to be involved in sleep regulation as well as pathophysiology of Tourette's syndrome. ${ }^{19-21}$ An unaltered serotonergic firing has been shown to be a prerequisite for sleep maintanance. ${ }^{22}$ Our finding of a significant positive correlation between Tourette's syndrome severity and features of disturbed sleep quality is compatible with the inverse correlation between tic severity and CSF concentrations of tryptophan, a precursor substance of serotonin. ${ }^{20}$ Both tics and insomnia of patients with Tourette's syndrome might be related to reduced serotonergic activity. Increased noradrenaline in patients with insomnia on the other hand is considered as a sign of hyperarousal $^{23}$ that has been found in Tourette's syndrome $^{20}$ as well. Together with increased CSF corticotropin releasing hormone concentrations in patients with Tourette's syndrome ${ }^{24}$ 
it may reflect similar psychophysiological hyperactivity in Tourette's syndrome.

In anology to the reciprocal interaction of reduced sleep quality and decreased daytime functioning in patients with primary insomnia ${ }^{25}$ we hypothesise a reciprocal interaction of reduced sleep quality and tic severity during the daytime in patients with Tourette's syndrome. This is consistent with the positive correlation between Tourette's syndrome severity and variables of sleep disturbance found in our study-that is, an increased Tourette's syndrome severity during the daytime being associated with a decreased sleep quality. Sleep with an increased number of awakenings as a sign of reduced sleep continuity is known to be less restorative. ${ }^{26}$ As with patients with insomnia $^{27}$ patients with Tourette's syndrome might be more irritable during the awake hours because of their reduced sleep quality. Triggered by this irritability they are prone to exhibit more tics in distressful situations. ${ }^{28}{ }^{29}$ The additional psychological strain induced by the need for coping with tics might maintain the hyperarousal. The hyperarousal in turn may affect the ability to initiate and maintain sleep making it less restorative, thereby closing the vicious circle of daytime tic intensity and sleep disturbance.

Because of the possible negative effects of insomnia on Tourette's syndrome symptomatology a disturbed sleep through tics should be taken into consideration in the planning of treatment strategies.

MOVEMENT ANALYSIS

The markedly disturbed sleep of patients with Tourette's syndrome was accompanied by increased motor activity in REM as well as non-REM sleep in comparison with healthy controls. This increased frequency of movements was not only due to the additional tics in patients with Tourette's syndrome but was also caused by an equally increased frequency of non-tic movements in patients with Tourette's syndrome. This can be interpreted as a sign of hyperarousal and is concordant with a reduced intracortical inhibition of motor pathways in Tourette's syndrome. ${ }^{30}$

The higher incidence of tics during REM sleep is paralleled by a similar increase in nontic movements of patients with Tourette's syndrome in this sleep stage. A comparable relation between movement frequency during REM sleep and non-REM sleep was found in our control subjects. A nearly $50 \%$ increase in movement frequency in REM sleep in comparison with non-REM sleep had been reported for healthy subjects in earlier work. ${ }^{31-34}$ Therefore similar mechanisms controlling the occurrence of tic and non-tic movements during this sleep stage are likely. In fact, the activity of all motor pathways is greatly enhanced in an apparently random manner during REM sleep $^{35}$ and might account for the higher movement frequency despite the markedly reduced muscle tone during this sleep stage.

In addition to the increased movement frequency the overall increased activity of motor pathways might be the source of the slight but significant increase in tic intensity during REM sleep in comparison with nonREM sleep. The absolute level of tic intensity, however, maintains to be mild both in REM as well as non-REM sleep. Most of the tics exhibited during REM and non-REM sleep were simple tics by contrast with the frequent occurence of complex tics during wakefulness. No difference in complexity of tics between REM and non-REM sleep was found. Unlike movement frequency tic intensity and tic complexity seemed to be largely uninfluenced by the type of sleep.

Often, movements during sleep are accompanied by short arousals. These EEG arousals during sleep reflect a brief cortical activation. Patients with Tourette's syndrome showed EEG arousals in conjunction with a movement during sleep significantly less often than healthy controls. This was mainly due to a reduced EEG arousal frequency in the temporal vicinity of tics. Therefore, tics seem to be accompanied by less cortical activity than regular movements. A corresponding aspect of the reduced cortical activity accompanying tics is the absence of a normal premovement EEG potential in awake patients with Tourette's syndrome before involuntary tics. ${ }^{36}$ These findings are consistent with the hypothesis that subcortical parts of the corticostriato-thalamocortical circuits play a major part in the pathophysiology of tics. ${ }^{28}$

Within the search for important factors in the pathophysiology of Tourette's syndrome there is a debate about the voluntary versus involuntary nature of tics. Sleep can be considered as a stage of extremely reduced or nearly absent voluntary control of cognition, emotion, and movement. Together with the occurrence of tics in all sleep stages the reduced EEG arousal frequency of tic movements is consistent with the hypothesis that involuntary aspects play a major part in the pathophysiology of tics.

1 Jankovic J, Rohaidy H. Motor, behavioral and pharmacologic findings in Tourette's syndrome. Can $\mathcal{F}$ Neurol Sci logic findings in
$1987 ; \mathbf{1 4}: 541-6$.

2 Comings DE, Comings BG. A controlled study of Tourette syndrome. VI. Early development, sleep problems, allergies, and handedness. Am $\mathcal{F}$ Hum Genet 1987;41:822-38.

3 Mendelson WB, Caine ED, Goyer P, et al. Sleep in Gilles de la Tourette syndrome. Biol Psychiatry 1980;15:339-43.

4 Glaze DG, Frost JD Jr, Jankovic J. Sleep in Gilles de la Tourette's syndrome: disorder of arousal. Neurology 1983; 33:586-92.

5 Voderholzer U, Müller N, Haag C, et al. Periodic limb movements during sleep are a frequent finding in patients with Gilles de la Tourette's syndrome. F Neurol 1997;244: 521-6.

6 Drake ME Jr, Hietter SA, Bogner JE, et al. Cassette EEG sleep recordings in Gilles de la Tourette syndrome. Clin Sleep recordings in Gilles de la

7 Moeller AA, Krieg JC. Sleep EEG in Gilles de la Tourette's syndrome. F Neurol 1992;239:113.

8 Tarajanovic NN, Shapiro CM, Sandor P. REM sleep behavTarajanovic NN, Shapiro CM, Sandor P. REM sleep behav-
iour disorder in patients with Tourette's syndrome. Sleep Research 1997;26:524.

9 Fish DR, Sawyers D, Allen PJ, et al. The effect of sleep on the dyskinetic movements of Parkinson's disease, Gilles de la Tourette syndrome, Huntington's disease, and torsion dystonia. Arch Neurol 1991;48:210-4

10 Singer HS. Neurobiology of Tourette syndrome. Neurol Clin 1997; 15:357-79.

11 Hobson JA, McCarley RW, Wyzynski PW. Sleep cycle oscillation: reciprocal discharge of two brainstem neuronal groups. Science 1975;189:55-8.

12 Shapiro AK, Shapiro ES, Young JG, et al. Gilles de la Tourette Syndrome. New York: Raven Press, 1988.

13 Rechtschaffen A, Kales A. A manual of standardized terminologie, techniques and scoring system for sleep stages of terminologie, techniques and scoring system for sleep stages of human subjects. Wash
Printing Office, 1968. 
14 Penzel T, Hajak G, Hoffmann RM, et al. Empfehlungen zur Durchführung und Auswertung polygraphischer Ableitungen im

15 ASDA. EEG arousals: scoring rules and examples, a preliminary report from the Sleep Disorders Atlas Task force of the American Sleep Disorders Association. Sleep 1992;15:174-83.

16 Leckman JF, Riddle MA, Hardin MT, et al. The Yale global tic severity scale: initial testing of a clinician-rated scale of tic severity. F Am Acad Child Adolesc Psychiatry 1989;28: 566-73.

17 Silvestri R, De Domenico P, Di Rosa AE, et al. The effect of nocturnal physiological sleep on various movement disorders. Mov Disord 1990;5:8-14.

18 Borbely AA. A two process model of sleep regulation. Hum Neurobiol 1982;1:195-204.

19 Adrien J. The serotonergic system and sleep-wakefulness regulation. In: Kales A, ed. Handbook of experimental pharregulation. In: Kales A, ed. Handbook of exp
macology. Berlin: Springer, 1995:91-116.

20 Leckman JF, Goodman WK, Anderson GM, et al. Cerebrospinal fluid biogenic amines in obsessive compulsive disorder, Tourette's syndrome, and healthy controls. Neuropsychopharmacology 1995;12:73-86.

21 Anderson GM, Pollak ES, Chatterjee D, et al. Brain monoamines and amino acids in Gilles de la Tourette's syndrome: a preliminary study of subcortical regions. Arch Gen Psychiatry 1992;49:584-6.

22 Jouvet M. Insomnia and decrease of cerebral 5-hydroxytryptamine after destruction of the raphe system in the cat. Adv Pharmacol 1968;6:265-79.

23 Adam K, Tomeny M, Oswald I. Physiological and psychological differences between good and poor sleepers. 7 Psychiatr Res 1986;20:301-16.

24 Chappell P, Leckman J, Goodman W, et al. Elevated cerebrospinal fluid corticotropin-releasing factor in Tourette's syndrome: comparison to obsessive compulsive disorder and normal controls. Biol Psychiatry 1996;39: disorder
25 Morin CM. Insomnia: psychological assessment and management. New York, London: Guildford Press, 1993.

26 Stepanski E, Lamphere J, Badia P, et al. Sleep fragmentation and daytime sleepiness. Sleep 1984;7:18-26.

27 Kales JD, Kales A, Bixler EO, et al. Biopsychobehavioral correlates of insomnia, V: Clinical characteristics and behavioral correlates. Am f Psychiatry 1984;141:1371-6.

28 Leckman JF, Peterson BS, Anderson GM, et al. Pathogenesis of Tourette's syndrome. $f$ Child Psychol Psychiatry 1997;38:119-42.

29 Licamele WL, Goldberg RL. Tourette syndrome. Am Fam Physician 1988;37:115-9.

30 Ziemann U, Paulus W, Rothenberger A. Decreased motor inhibition in Tourette's disorder: evidence from transcranial magnetic stimulation. Am $\mathcal{F}$ Psychiatry 1997;154:127784.

31 Wilde-Frenz J, Schulz H. Rate and distribution of body movements during sleep in humans. Percept Mot Skills 1983;56:275-83.

32 Naitoh P, Muzet A, Johnson LC, et al. Body movements during sleep after sleep loss. Psychophysiology 1973;10:363-

33 Williams HL, Williams CL. Nocturnal EEG profiles and performance. Psychophysiology 1966;3:164-75.

34 Dement W, Kleitman N. Cyclic variations in EEG during sleep and their relation to eye movements, body motility, and dreaming. Electroencephalogr Clin Neurophysiol 1957;3: 673-90.

35 Chase MH, Morales FR. The control of motoneurons during sleep. In: Kryger MHR, Dement WC, eds. Principles and practice of sleep medicine, 2nd ed. Philadelphia: WB Saunders, 1994:163-75.

36 Obeso JA, Rothwell JC, Marsden CD. Simple tics in Gilles de la Tourette's syndrome are not prefaced by a normal premovement EEG potential. F Neurol Neurosurg Psychiatry 1981;44:735-8. 